\title{
Some cross-cultural data on probability learning'
}

\author{
MICHAEL COLE, YALE UNIVERSITY LEO KELLER, STANFORD UNIVERSITY
} NINA N. KORZH, MOSCOW STATE UNIVERSITY

Extended training in a probability learning task was given to $17 \mathrm{Ss}$ from two non-western cultures. Ss came from Moscow University, USSR and Cuttington, Liberia, a small rural African town. Long-term probability matching was obtained in both groups. However, the trial-to-trial changes in response proportions varied markedly between groups; Liberian $S s$ tended to follow the reinforcing events, while responses by the Russian Ss depended more heavily on their own preceding responses. Some negative recency effect was obtained for both groups. This effect decreased somewhat over trials, but was still present at the termination of training.

A probability learning task was run for an extended number of short sessions on two groups of Ss from non-Western cultures. Most of the previous studies have used Ss from the West European-American cultural milieu and the typical results for group data are probability matching (see Estes, 1964) and negative recency (see Jarvik, 1951; Nicks, 1959). Probability matching occurs when the mean learning curve approaches the probability of the occurrence of the predicted event, and negative recency occurs when the probability of predicting an event decreases as the runs of that event increase.

\section{Subjects}

\section{METHOD}

The first group consisted of seven college students from Moscow State University. The second group was 16 schoolboys from small villages in Liberia. They were 10 to 14 years old and had one to two years of formal education.

Procedure

A sequence of reinforcing events was generated separately for each $\mathrm{S}$ using a method of unrestricted randomization with the probability of event "1" equal to .667 and the probability of event "2"' equal to .333 . There were 100 trials which took about $6 \mathrm{sec}$. each in a session. Sessions ordinarily followed each other by at least a 1-day interval, although on occasion several days intervened between sessions. Due to language difficulties and time schedules the following procedural differences occurred: (1) The Russian group responded verbally to E's signal of tapping a pencil on the table, and then $E$ read aloud the appropriate event. Twenty sessions were run. (2) The Liberian group predicted whether one or two bottle tops would be shown, and $\mathrm{E}$ produced one or two of them. Five sessions were run for $10 \mathrm{Ss}$ while additional $6 \mathrm{Ss}$ completed two sessions. The data from all Ss are used where appropriate.

\section{General Findings}

\section{RESULTS}

Figure 1 shows the mean probability of choosing the more frequently reinforced alternative in 50-trial

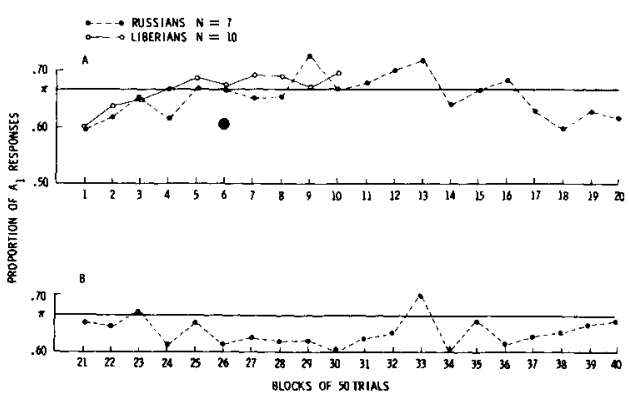

Fig. 1. Learning curves

blocks. For the 500 trials they have in common, the curves of the two groups are very much alike. With more extended trials, the Russian group's curve is relatively stable just below the event probability value. The terminal mean response proportions in Table 1 indicate that none of the Ss are maximizing (consistently choosing the more frequently reinforced event) and that several Ss are very close to the matching level.

Recency analysis

The recency curves plotted in Fig. 2 a-b and Fig. $3 \mathrm{a}-\mathrm{b}$ show the proportion of predicting that an event will occur as a function of the length of the immediately preceding run of that particular event. The analysis has been divided into three blocks of trials in order to investigate possible changes in the shapes of the curves as a function of training. Although the data manifest some variability, certain trends may be discerned. Most of the curves decrease as the length of the preceding runs becomes longer. There are, however, some portions which increase with increasing runs. There is also a tendency for the curves to reach and maintain a higher level as the amount of training increases.

The recency curves of the Liberian group differ from those of the Russian group in two distinct ways. First,

Table 1. Terminal Proportions of $A$, Choices by Subjects

\begin{tabular}{|c|c|c|}
\hline \multirow[b]{2}{*}{ Subject } & \multicolumn{2}{|c|}{ Group } \\
\hline & Russian ${ }^{1}$ & Liberian 2 \\
\hline 1 & .599 & .768 \\
\hline 2 & .557 & .680 \\
\hline 3 & .673 & .796 \\
\hline 4 & .671 & .572 \\
\hline 5 & .644 & .800 \\
\hline 6 & .667 & .512 \\
\hline 7 & .698 & .668 \\
\hline 8 & - & .720 \\
\hline 9 & - & .608 \\
\hline 10 & - & .740 \\
\hline
\end{tabular}

1 Trials 1001-2000

2 Trials $376-500$ 


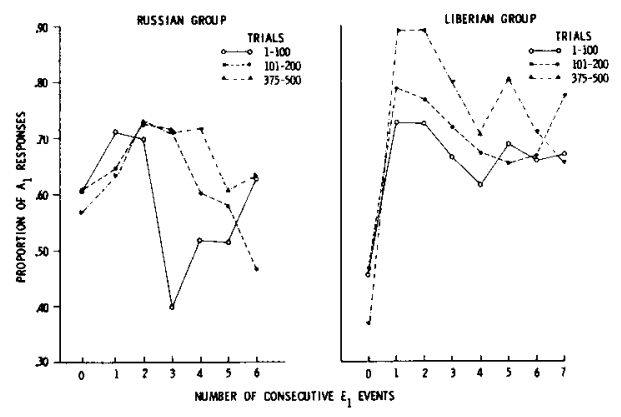

Fig. 2, Recency curves for the most frequent event

the Liberians show a strong tendency to switch their predictions following a change in the event, while the Russians do not. Secondly, the Liberian curves for the $\mathrm{A}_{2}$ response following 1,2 , and 3 preceding occurrences of $\mathrm{E}_{2}$ are at a much higher level than the overall level of making the $A_{2}$ response, while the Russian curves remain close to the overall level of choosing $\mathrm{A}_{2}$.

Sequential analysis

The proportion of responses conditional on the previous response and the previous event are presented in Table 2 for the final blocks of trials. The Liberian group shows a tendency to predict the event which occurred on the previous trial, but with relatively little dependence on the previous response. The Russian

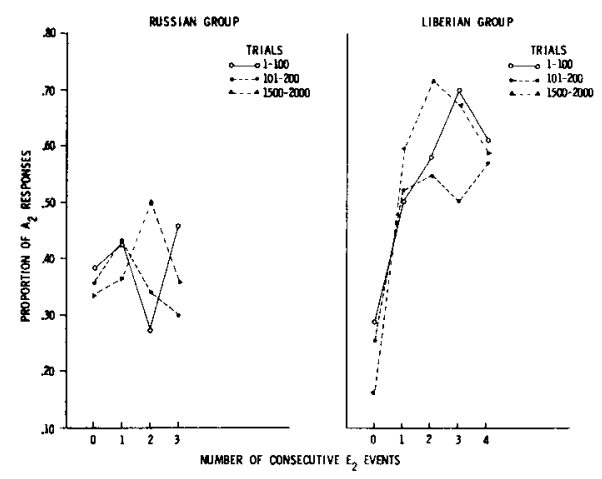

Fig. 3. Recency curves for the least frequent event
Table 2. Conditional Proportions for a Terminal Block of Trials

\begin{tabular}{l|ccc} 
Event & Liberians & Russians $^{2}$ \\
\hline $\mathrm{A}_{1, \mathrm{n}+1}$ & $\mathrm{E}_{1, \mathrm{n}}$ & .84 & .67 \\
$\mathrm{~A}_{1, \mathrm{n}+1}$ & $\mathrm{E}_{2, \mathrm{n}}$ & .35 & .62 \\
$\mathrm{~A}_{1, \mathrm{n}+1}$ & $\mathrm{~A}_{1, \mathrm{n}}$ & .68 & .68 \\
$\mathrm{~A}_{1, \mathrm{n}+1}$ & $\mathrm{~A}_{2, \mathrm{n}}$ & .71 & .56 \\
\hline
\end{tabular}

1 Trials $376-500$

2 Trials 1501-2000

group shows a different pattern with little dependence on the previous event, but a slight tendency to repeat the previous response.

\section{DISCUSSION}

Although both groups showed evidence of a negative recency effect, none of the curves conform to the classic form of the negative recency curve as found by Jarvik (1951) and Nicks (1959). Some changes toward less negative recency occur during the training period but even in the final block of trials the curves are not monotonically increasing.

In view of the unavoidable multiple confoundings inherent in our casual data gathering procedures, we can only indicate what we view as the more reliable conclusions suggested by the data. First, there is evidence of long term probability matching by both groups. Second, there is an unusually strong tendency for the Liberian group to predict the event which occurred on the previous trial. Third, there is the presence of negative recency or "gambler's fallacy" in two non-American cultures.

\section{References}

Estes, W. K. Probability learning. In A. W. Melton (Ed.), Categories of human learning. New York: Academic Press, 1964. Pp. 89-128. Jarvik, M. E. Probability learning and a negative recency effect in the serial anticipation of alternative symbols. J. exp. Psychol., 1951, 41, 291-297.

Nicks, D. C. Prediction of sequential two-choice decisions from event runs. $J$. exp. Psychol., 1959, 57, 105-114.

\section{Note}

1. We acknowledge the support and cooperation of the Governments of the United States and Soviet Union, Educational Service, Inc. Cuttington Wollege. Preparation of this report was supported in part by O.N.R. Contract Nonr. 225(73). Thanks are due W. K. Estes for his comments on the research. 